\title{
Developing a Diagnostic CT Scoring System for Granulomatous-Lymphocytic Interstitial Lung Disease (GLILD) in Common Variable Immunodeficiency Patients
}

Stella Hartono ( $\nabla$ stella.hartono@nih.gov )

Baylor College of Medicine https://orcid.org/0000-0002-2276-113X

Christian W. Cox

Mayo Clinic, Rochester

Rebecca M Lindell

Mayo Clinic, Rochester

Jonathan $\mathrm{H}$. Chung

University of Chicago Medicine

Avni Y. Joshi

Mayo Clinic, Rochester

\section{Research Article}

Keywords: Common variable immunodeficiency (CVID), granulomatous lymphocytic interstitial lung disease (GLILD), scoring systems, lung biopsy

Posted Date: April 19th, 2021

DOI: https://doi.org/10.21203/rs.3.rs-406317/v1

License: (c) (i) This work is licensed under a Creative Commons Attribution 4.0 International License.

Read Full License 


\section{Abstract}

\section{Purpose}

To determine the utility of GLILD CT scoring system for assessment of GLILD in patients with CVID to avoid the need for lung biopsy.

\section{Methods}

The CT scoring system was developed as a consensus based on review of literature. Two radiologists, blinded to patient clinical information, retrospectively scored CT scans of selected patients with histopathologic diagnosis of GLILD. Discrepancies were settled by a third radiologist. Inter-observer reproducibility was calculated with intra-class correlation coefficients.

\section{Results}

We identified 25 CVID patients with histologically confirmed GLILD (12 male, 13 female). The median age was 38 years (range 12 - 72 years). Inter-observer reproducibility was 0.76 (95\% $\mathrm{Cl} 0.53-0.89$ ). Splenomegaly/splenectomy and basilar predominant perilymphatic nodules greater than or equal to $4 \mathrm{~mm}$ have good interobserver agreement in our CT scoring system. Discrepancy between observers occurred most often in differentiating intermediate versus high confidence diagnosis of GLILD as opposed to differentiating between low versus intermediate confidence.

Conclusion

GLILD CT scoring system of pulmonary disease in CVID patients can stratify patients into categories that may assist in avoiding lung biopsy for diagnosis in a subset of patients.

\section{Introduction}

Common variable immunodeficiency disorders (CVID) are a group of heterogeneous diseases primarily characterized by hypogammaglobulinemia and increased risk of infection. As treatment with immunoglobulin replacement therapy has increased median survival, noninfectious complications have become the predominant source of morbidity and mortality in these patients (1-3). Depending on the study, $8 \%$ to $22 \%$ of people living with CVID develop an interstitial lung disease (ILD) termed "granulomatous-lymphocytic interstitial lung disease" (GLILD) (4-8) which has been associated with early mortality in patients with CVID (5).

Over ten years ago it was established by Routes and colleagues that the presence of GLILD significantly worsens survival in CVID. However, GLILD has proven challenging to diagnose, difficult to treat, and is not reversed by conventional immunoglobulin (Ig) replacement therapy in most patients. New treatment using immunomodulation, especially the combination of rituximab and azathioprine has shown promise (6); however, lung disease may return or continue to progress when treatment is terminated. 
The pathogenesis of GLILD in CVID is not well understood. While most pulmonary manifestations of CVID represent recurrent infections with up to $50 \%$ developing bronchiectasis, not all have or will progress to GLILD. Currently, confirmatory diagnosis of GLILD requires lung biopsy, which is an invasive procedure with substantial risk and complications. It is relatively common for CVID patients to have lung structural abnormalities on their HRCT, likely due to frequent respiratory infections (9-11). Indeed, comparison of chest $x$-rays (CXR), pulmonary function test (PFT) and high resolution computed tomography (HRCT) showed HRCT as the most sensitive method for identification of structural abnormalities, detecting pulmonary complications that were missed on CXR and PFT in 2-59\% of patients (12). Scoring systems based on HRCT have been commonly used to diagnose and track progression of various lung diseases such as cystic fibrosis $(13-16)$, sarcoidosis $(17,18)$, tuberculosis $(19)$, and idiopathic pulmonary fibrosis (IPF) $(20,21)$. A CT scoring system for GLILD may offer a reliable alternative to biopsy in a subset of CVID patients demonstrating a classic CT appearance of GLILD and may provide prognostic value in determining treatment. Therefore, we propose and evaluate a potential CT scoring system to initially diagnose GLILD and test it retrospectively in patients with confirmed diagnosis of GLILD in order to determine reproducibility between radiologists and refine the scoring system for future studies.

\section{Materials And Methods}

Study Population Following IRB approval, a retrospective chart review of the electronic database was conducted to identify CVID patients with GLILD who underwent chest CT between March 2013 to October 2016 as part of the routine clinical protocol to evaluate GLILD. GLILD diagnosis was confirmed by histopathologic evidence of granulomatous disease in the lungs or alternative organ(s) in the setting of CVID and CT proven interstitial lung disease. The CT at time of GLILD diagnosis was used for scoring purposes. All patients were evaluated at Mayo Clinic, Rochester, MN. Proposed GLILD scoring system on Chest CT The identification of variables to be included in the analysis was based on a consensus of three board certified radiologist specializing in thoracic radiology and guided by a review of the literature (2227). Features visualized on the chest imaging suggestive of GLILD that were consistent across the reviewed literature included the presence of perilymphatic pulmonary nodules, septal thickening in the lung bases, patchy bilateral ground glass opacities, thoracic lymphadenopathy (LAN), and splenomegaly. Imaging features of perilymphatic nodularity and perivascular cysts were included to reflect the known overlap in appearances with sarcoidosis and lymphocytic interstitial pneumonia, respectively. Nodules that surround patent airways were included based on anecdotal experience of the radiologists. We categorized these features into two groups: pulmonary nodule/nodularity scoring systems in which a score of 1 to 3 is assigned based on size and distribution patterns of the pulmonary nodules, and a score of 1 for presence of other abnormalities listed above. Representative CT images of typical GLILD CT patterns are provided in Figure 1. The total scores were derived as composites of all scored variables. The scoring system proposed is summarized in Table 1. In the setting of a clinical diagnosis of CVID, we prospectively set a score of 5 or more as high confidence for radiologic diagnosis of GLILD, score of 3-4

as intermediate confidence for radiologic diagnosis of GLILD, and score of 2 or less as low confidence for radiologic diagnosis of GLILD. Chest CT Acquisition Of the 25 identified patients, 21 underwent high 
resolution helical chest CT in supine inspiration at our institution with reconstructed images at $1.5 \mathrm{~mm}$ slice thickness. "High resolution" chest CT was defined for the study as less than $2 \mathrm{~mm}$ slice thickness in a high spatial resolution kernel as per Fleischner Society Diagnostic Criteria for Idiopathic Pulmonary Fibrosis (28). The remaining four CT exams were performed at outside institutions utilizing variable equipment and acquisitions to include two high-resolution chest CT studies, one pulmonary CT angiography, and one standard chest CT at $2 \mathrm{~mm}$ slice thickness. Eight chest CT exams were performed with IV contrast. Evaluation of Chest CT Blinded to clinical information beyond the study inclusion criteria, two independent board-certified fellowship trained thoracic radiologists (CWC and RML with 12 and 18 years of experience, respectively) evaluated each CT and assigned scores based on the proposed scoring system. Images were reviewed on GE Centricity RA 1000 version 4.1 PACS with EIZO RadiForce RX340 diagnostic monitors and scoring recorded on a computerized form. Following completion of scoring, the evaluators also provided subjective assessment regarding expected appearance of GLILD as well as non-scored findings. In this study, discrepancies between the primary evaluators were resolved through a tie-breaker score provided by a third board certified fellowship trained thoracic radiologist (JHC with 10 years of experience). Statistical Analysis Interobserver reproducibility of the scoring systems was tested with the intraclass correlation coefficient (Ri) and Bland-Altman plot. Values of Ri greater than 0.80 are generally considered to represent good agreement between observers. Interobserver agreement of the various scoring system variables was evaluated with the statistic. The values of $0.20,0.21-0.40,0.41-$ $0.60,0.61-0.80$, and $0.81-1.00$ are generally considered to represent poor, fair, moderate, good, and very good agreement, respectively. Radiologic diagnoses based on the scoring system were then compared to histopathology diagnosis using Spearman rank coefficient. A software package (JMP Pro version 13) was used for statistical analysis. A difference with a $\mathrm{P}$ value less than .05 was considered significant.

\section{Results}

\section{Study Population}

Table 2 summarizes the distribution of the study population. We identified 25 CVID patients with GLILD (12 male patients, 13 female patients). The median age was 38 years (range 12 - 72 years old) at the time of CT imaging. GLILD was confirmed by histopathology analysis of lung or biopsy in 21 of the patients. Four of the patients were diagnosed by biopsy of granulomas found in the spleen or lymph nodes in the setting of interstitial lung disease proven by CT. Clinical and immunological characteristics of these patients are summarized in Table 2. Eight patients were current smokers while the other 17 patients never smoked.

\section{Thin-Section CT Scoring Systems}

When considering radiologic findings identified by at least one thoracic radiologist, the most common CT finding in the study cohort was splenomegaly or splenectomy $(n=20,80 \%)$. Other common CT findings identified by at least one observer included pulmonary nodules surrounding patent airways ( $n=16,64 \%)$ and bibasilar predominant perilymphatic nodules equal to or greater than $4 \mathrm{~mm}(\mathrm{n}=14,56 \%)$. (Table 3$)$ 
When considering agreement between the two primary thoracic radiologists for a given radiologic finding, splenomegaly/splenectomy remained the most common finding $(n=17)$, and bibasilar predominant perilymphatic nodules equal to or greater than $4 \mathrm{~mm}$ became the second most common finding $(n=10)$.

With regards to uncommon CT findings, bilateral perilymphatic nodularity less than $4 \mathrm{~mm}$ was only considered present in one patient by one thoracic radiologist. Pulmonary cysts were not identified by any reviewers.

Interobserver variability was generally good (Ri $0.76,95 \% \mathrm{Cl} 0.53-0.89$ ) (Table 3). Agreement was good in rating of bibasilar perilymphatic nodules equal to or greater than $4 \mathrm{~mm}$, bilateral perilymphatic nodularity less than $4 \mathrm{~mm}$, and splenomegaly/splenectomy. However, coefficients were less than 0.61 for most scoring system variables. For bilateral perilymphatic nodules without basilar predominance equal to or greater than $4 \mathrm{~mm}$, septal thickening in the bases, LAN, and chronic bilateral groundglass/cysts, coefficients were less than 0.40 for all comparisons.

\section{Predictive value of the CT scoring systems}

From 25 cases evaluated by the CT scoring system, 12 cases (48\%) were categorized as high confidence GLILD, 3 cases (12\%) diagnosed as intermediate confidence GLILD, and 10 cases (40\%) diagnosed as low confidence for GLILD. (Table 3) The Bland-Altman plot (Figure 2) illustrates that there was no particular bias between the two reviewers. The main disagreement between the reviewers were in differentiating the intermediate vs. high confidence diagnosis of GLILD.

\section{Discussion}

This study attempts to develop and test a systematic scoring system that can assist clinicians and radiologists in evaluation of GLILD in patients with CVID using Chest CT. Based on our systematic review of the literature and experience, our proposed scoring system included classic findings of GLILD as well as potential characteristic patterns typical of sarcoidosis (perilymphatic nodularity) and lymphoproliferative disease (perilymphatic cysts). Consistent with the findings of Mannina et al, splenomegaly and splenectomy were the most common finding in CVID related GLILD [25], with our study supporting this as a reproducible finding between observers. Bibasilar predominant perilymphatic nodules greater than or equal to $4 \mathrm{~mm}$ were weighted heavily to reflect the characteristic finding in GLILD and proved to have good agreement between radiologists, favoring that this finding should be kept as a central finding in GLILD CT scoring. Nodules surrounding patent airways is a common finding associated with GLILD, although our study suggests that characterization of such nodules may be inconsistent between thoracic radiologists. Bilateral perilymphatic nodularity less than $4 \mathrm{~mm}$ and perilymphatic cysts, more characteristic of sarcoidosis and lymphocytic interstitial pneumonia respectively, were uncommon findings and may not contribute meaningfully to the scoring system.

Certain CT imaging findings commonly seen in CVID were purposefully excluded as they suggest alternative diagnoses that are not mutually exclusive from GLILD. Bronchiectasis in the lower lungs is a 
common finding in CVID related lung disease, although is attributable to immunodeficiency and recurrent infection. Alternatively, mass-like opacities or consolidation are not characteristic of GLILD and raise concern for complicating lymphoma, particularly when chronic (29).

Our novel scoring system may have many advantages. Firstly, it has the potential to help standardize and streamline the diagnosis of GLILD. A community radiologist, who is not familiar with GLILD, may be able to use this scoring system with the provided example images to help make an early diagnosis, which so far has been left to individual features without a conclusive system to help further management. Secondary, this may improve overall patient management and be cost saving. When the score is in the range of "high confidence for GLILD", as nearly half the patients in our study were, additional invasive interventions like an open lung biopsy may be able to be avoided. Some radiologists may score confident cases as intermediate or low confidence for GLILD and, in those cases, a second radiology opinion, interdisciplinary conference discussion and/or additional interventions may be pursued.

Overall, the inter-observer variability based on these criteria is good, and most of the disagreement is in differentiating intermediate vs high confidence diagnosis of GLILD instead of differentiating between low vs intermediate or high confidence. Category descriptors are critical in defining downstream decisions, and alternative category names could be considered, such as "consistent with", "probable" and "inconclusive for" GLILD. Such categories could then favor biopsy in patients with "inconclusive" CT findings, favor avoiding biopsy in "consistent with" CT findings and favor multidisciplinary discussion for consideration of biopsy in "probable/possible" findings. While our scoring system only captured $60 \%$ of the GLILD confirmed cohort when scored across multiple radiologists, these patients could have potentially avoided the risk of biopsy by meeting imaging criteria for GLILD, much like how diagnostic criteria for IPF define the usual interstitial pneumonia CT pattern to avoid biopsy $(20,21)$.

Primary limitations of our study include the retrospective design, limited number of patients, absence of control group, variability of CT imaging during the time course of disease, absent surveillance imaging to assess evolution of disease, and cohort selection from a quaternary referral center. At the same time, the cohort was relatively large for the rarity of GLILD and did reflect our experience of the scope of GLILD CT findings. While the scoring did not represent the evolution of disease over time, the scoring of a single timepoint at initial diagnosis is more representative of general practice and the intended application of the scoring system.

Future considerations for study should focus on prospective application of CT scoring of possible GLILD in practice, to include determining sensitivity and specificity when compared to histopathologic results. Additional potential considerations include refinement of scoring by inclusion of alternative laboratory or physiologic testing (30), the relationship of prognosis to CT scoring and the potential for CT scoring in treatment decision making.

In conclusion, splenomegaly/splenectomy and basilar predominant perilymphatic nodules greater than $4 \mathrm{~mm}$ are typical CT findings of GLILD and have good interobserver agreement in a CT scoring system. Bilateral perilymphatic nodularity less than $4 \mathrm{~mm}$ and perivascular cysts can likely be excluded from CT 
scoring of GLILD aimed at identifying characteristic findings of GLILD but may need further validation using a larger prospective cohort. Finally, a GLILD CT scoring system of pulmonary disease in CVID patients can help in early identification and stratification of patients that may assist in clinical management and potentially avoid invasive lung biopsy for diagnosis.

\section{Declarations}

\section{Compliance with ethical standards}

This study was approved by the university human research ethics committee and all procedures performed in studies involving human participants were in accordance with the ethical standards of the institutional and/or national research committee and with the 1964 Helsinki declaration and its later amendments or comparable ethical standards. Informed consent was obtained from all individual participants included in the study.

\section{Authors Contribution}

SPH participated in the design of the study, collected and interpreted the data, and draft the manuscript. RML and JHC participated in the design, conducted the study, and helped to draft the manuscript. CWC and AYJ conceived of the study, formulated its design, coordinated the conduct of the study including data collection, helped to interpret the data and helped to draft the manuscript. All authors read and approved the final manuscript.

\section{Funding}

No funding was received for conducting this study.

\section{Competing interest}

The authors declare no conflict of interest

\section{Availability of data and materials}

The datasets generated during and/or analyzed during the current study are available from the corresponding author on reasonable request

\section{Disclosure of conflict of interest:}

The authors declare no conflict of interest. The authors received no specific funding for this work.

\section{References}

1. Chapel H, Lucas M, Lee M, Bjorkander J, Webster D, Grimbacher B, et al. Common variable immunodeficiency disorders: division into distinct clinical phenotypes. Blood. 2008;112(2):277-86. 
2. Orange JS, Grossman WJ, Navickis RJ, Wilkes MM. Impact of trough IgG on pneumonia incidence in primary immunodeficiency: A meta-analysis of clinical studies. Clin Immunol. 2010;137(1):21-30.

3. Resnick ES, Moshier EL, Godbold JH, Cunningham-Rundles C. Morbidity and mortality in common variable immune deficiency over 4 decades. Blood. 2012;119(7):1650-7.

4. Park JH, Levinson Al. Granulomatous-lymphocytic interstitial lung disease (GLILD) in common variable immunodeficiency (CVID). Clin Immunol. 2010;134(2):97-103.

5. Bates CA, Ellison MC, Lynch DA, Cool CD, Brown KK, Routes JM. Granulomatous-lymphocytic lung disease shortens survival in common variable immunodeficiency. J Allergy Clin Immunol. 2004;114(2):415-21.

6. Chase NM, Verbsky JW, Hintermeyer MK, Waukau JK, Tomita-Mitchell A, Casper JT, et al. Use of combination chemotherapy for treatment of granulomatous and lymphocytic interstitial lung disease (GLILD) in patients with common variable immunodeficiency (CVID). J Clin Immunol. 2013;33(1):309.

7. Morimoto Y, Routes JM. Granulomatous disease in common variable immunodeficiency. Curr Allergy Asthma Rep. 2005;5(5):370-5.

8. Maarschalk-Ellerbroek LJ, de Jong PA, van Montfrans JM, Lammers JW, Bloem AC, Hoepelman Al, et al. CT screening for pulmonary pathology in common variable immunodeficiency disorders and the correlation with clinical and immunological parameters. J Clin Immunol. 2014;34(6):642-54.

9. Cunningham-Rundles $C$, Bodian C. Common variable immunodeficiency: clinical and immunological features of 248 patients. Clin Immunol. 1999;92(1):34-48.

10. Feydy A, Sibilia J, De Kerviler E, Zagdanski AM, Chevret S, Fermand JP, et al. Chest high resolution CT in adults with primary humoral immunodeficiency. Br J Radiol. 1996;69(828):1108-16.

11. Thickett KM, Kumararatne DS, Banerjee AK, Dudley R, Stableforth DE. Common variable immune deficiency: respiratory manifestations, pulmonary function and high-resolution CT scan findings. QJM. 2002;95(10):655-62.

12. Touw CM, van de Ven AA, de Jong PA, Terheggen-Lagro S, Beek E, Sanders EA, et al. Detection of pulmonary complications in common variable immunodeficiency. Pediatr Allergy Immunol. 2010;21(5):793-805.

13. Bhalla M, Turcios N, Aponte V, Jenkins M, Leitman BS, McCauley DI, et al. Cystic fibrosis: scoring system with thin-section CT. Radiology. 1991;179(3):783-8.

14. Brody AS, Molina PL, Klein JS, Rothman BS, Ramagopal M, Swartz DR. High-resolution computed tomography of the chest in children with cystic fibrosis: support for use as an outcome surrogate. Pediatr Radiol. 1999;29(10):731-5.

15. Nathanson I, Conboy K, Murphy S, Afshani E, Kuhn JP. Ultrafast computerized tomography of the chest in cystic fibrosis: a new scoring system. Pediatr Pulmonol. 1991;11(1):81-6.

16. Santamaria F, Grillo G, Guidi G, Rotondo A, Raia V, de Ritis G, et al. Cystic fibrosis: when should highresolution computed tomography of the chest Be obtained? Pediatrics. 1998;101(5):908-13. 
17. Drent M, De Vries J, Lenters M, Lamers RJ, Rothkranz-Kos S, Wouters EF, et al. Sarcoidosis: assessment of disease severity using HRCT. Eur Radiol. 2003;13(11):2462-71.

18. Oberstein A, von Zitzewitz H, Schweden F, Muller-Quernheim J. Non invasive evaluation of the inflammatory activity in sarcoidosis with high-resolution computed tomography. Sarcoidosis Vasc Diffuse Lung Dis. 1997;14(1):65-72.

19. Ors F, Deniz O, Bozlar U, Gumus S, Tasar M, Tozkoparan E, et al. High-resolution CT findings in patients with pulmonary tuberculosis: correlation with the degree of smear positivity. J Thorac Imaging. 2007;22(2):154-9.

20. Jacob J, Bartholmai BJ, Rajagopalan S, Kokosi M, Nair A, Karwoski R, et al. Automated Quantitative Computed Tomography Versus Visual Computed Tomography Scoring in Idiopathic Pulmonary Fibrosis: Validation Against Pulmonary Function. J Thorac Imaging. 2016;31(5):304-11.

21. Jacob J, Bartholmai BJ, Rajagopalan S, Kokosi M, Egashira R, Brun AL, et al. Serial automated quantitative CT analysis in idiopathic pulmonary fibrosis: functional correlations and comparison with changes in visual CT scores. Eur Radiol. 2018;28(3):1318-27.

22. Maglione PJ, Overbey JR, Radigan L, Bagiella E, Cunningham-Rundles C. Pulmonary radiologic findings in common variable immunodeficiency: clinical and immunological correlations. Ann Allergy Asthma Immunol. 2014;113(4):452-9.

23. Torigian DA, LaRosa DF, Levinson Al, Litzky LA, Miller WT, Jr. Granulomatous-lymphocytic interstitial lung disease associated with common variable immunodeficiency: CT findings. J Thorac Imaging. 2008;23(3):162-9.

24. Bouvry D, Mouthon L, Brillet PY, Kambouchner M, Ducroix JP, Cottin V, et al. Granulomatosisassociated common variable immunodeficiency disorder: a case-control study versus sarcoidosis. Eur Respir J. 2013;41(1):115-22.

25. Mannina A, Chung JH, Swigris JJ, Solomon JJ, Huie TJ, Yunt ZX, et al. Clinical Predictors of a Diagnosis of Common Variable Immunodeficiency-related Granulomatous-Lymphocytic Interstitial Lung Disease. Ann Am Thorac Soc. 2016;13(7):1042-9.

26. Gregersen S, Aalokken TM, Mynarek G, Kongerud J, Aukrust P, Froland SS, et al. High resolution computed tomography and pulmonary function in common variable immunodeficiency. Respir Med. 2009;103(6):873-80.

27. Park JE, Beal I, Dilworth JP, Tormey V, Haddock J. The HRCT appearances of granulomatous pulmonary disease in common variable immune deficiency. Eur J Radiol. 2005;54(3):359-64.

28. Lynch DA, Sverzellati N, Travis WD, Brown KK, Colby TV, Galvin JR, et al. Diagnostic criteria for idiopathic pulmonary fibrosis: a Fleischner Society White Paper. Lancet Respir Med. 2018;6(2):13853.

29. Sirajuddin A, Raparia K, Lewis VA, Franks TJ, Dhand S, Galvin JR, et al. Primary Pulmonary Lymphoid Lesions: Radiologic and Pathologic Findings. Radiographics. 2016;36(1):53-70.

30. Hartono S, Motosue MS, Khan S, Rodriguez V, lyer VN, Divekar R, et al. Predictors of granulomatous lymphocytic interstitial lung disease in common variable immunodeficiency. Ann Allergy Asthma 
Immunol. 2017;118(5):614-20.

\section{Tables}

Table 1. Proposed GLILD Grading on Chest CT:

\begin{tabular}{|l|c|}
\hline Description / Conditions & Score \\
\hline PULMONARY NODULES: (select primary pattern) & 3 \\
\hline Bibasilar predominant perilymphatic nodules (= or $>4 \mathrm{~mm}^{*}$ & 1 \\
\hline Bilateral perilymphatic nodules without basilar predominance $(=$ or $>4 \mathrm{~mm})$ & 1 (S) \\
\hline Bilateral perilymphatic nodularity $(<4 \mathrm{~mm})$ & 1 \\
\hline ADDITIONAL CT FINDINGS: (select all that apply) & 1 \\
\hline Pulmonary nodules surround patent airways & 1 (L) \\
\hline Pulmonary septal thickening in the lung bases & 1 \\
\hline $\begin{array}{l}\text { Patchy bilateral chronic ground glass* opacities or scattered perivascular cysts as the } \\
\text { predominant pattern }\end{array}$ & 1 \\
\hline Thoracic lymphadenopathy (> 1.5 cm in short axis) & 10 \\
\hline Splenomegaly or splenectomy & \\
\hline TOTAL POSSIBLE SCORES & $\geq 5$ \\
\hline In the setting of a clinical diagnosis of CVID & $3-4$ \\
\hline High confidence of GLILD radiologic diagnosis & $\leq 2$ \\
\hline Intermediate confidence of GLILD radiologic diagnosis & \\
\hline Low confidence of GLILD radiologic diagnosis & \\
\hline
\end{tabular}

* Nodules greater than $1.5 \mathrm{~cm}$, nodules growing over time, masses or chronic consolidation should be evaluated for lymphoma

${ }^{\star \star}$ Chronic defined as persisting longer than 3 months without immunosuppressive treatment

Table 2 - Patients Characteristics 
Demographics

Age (year), range

$38.9 \pm 15.1,12-71$

Female sex, $\mathrm{n}(\%)$

$13(52 \%)$

Cigarette smoking status

Current smoker, $\mathrm{n}(\%)$

$8(32 \%)$

Never smoker, n (\%)

$17(68 \%)$

Extrapulmonary manifestations

Cytopenia

$7(36 \%)$

Splenomegaly/splenectomy

$17(68 \%)$

Granulomatous hepatitis

$7(36 \%)$

Time from initial CVID diagnosis to CT evaluation (year), range $3.5 \pm 4.7(0-16.7)$

Pulmonary function tests, \% predicted

FVC (Forced Vital Capacity (L)

$3.8(3.3-4.29)$

FEV1 (Forced Expiratory Volume in $1 \mathrm{sec}$ ) \%

$85 \pm 17$

FEV1/FVC ratio

$76 \pm 12.3$

DLCO

$73.2 \pm 20.3$

IgG dosage ( $\mathrm{mg} / \mathrm{kg} / \mathrm{month})$

$587.3 \pm 187$

Trough > 1000, n (\%)

$13(59 \%)$

Serum immunoglobulin

$\operatorname{lgG}(767-1590 \mathrm{mg} / \mathrm{dl})$

$257 \pm 188$

$\operatorname{lgA}(61-356 \mathrm{mg} / \mathrm{dl})$

$17 \pm 27.7$

$\operatorname{lgM}(37-286 \mathrm{mg} / \mathrm{dl})$

$33.6 \pm 23.2$

Peripheral blood lymphocytes, cells/mcL\% (95\% mean)

CD3 T cells

1101.8 (716-1487)

CD4 T cells

$545.3(420.9-669.5)$

CD8 T cells

$572.4(236.9-880.8)$

CD4/CD8 ratio

$1.8(1.1-2.4)$

CD19 B cells

$95.1(43-146)$

B-cell subsets, \% 


\begin{tabular}{|ll|}
\hline $\operatorname{lgD}+\mathrm{CD} 27+$ B cells & $6.4 \pm 5.5$ \\
\hline $\operatorname{lgD}-\mathrm{CD} 27+$ B cells & $2.9 \pm 7.2$ \\
\hline $\operatorname{lgM}+$ CD19+ B cells & $74 \pm 16$ \\
\hline
\end{tabular}

Table 3 - CT Scoring values for radiologic diagnosis of GLILD.

\begin{tabular}{|c|c|c|c|c|}
\hline Radiologic Finding & $\begin{array}{l}\text { Agreement between } \\
\text { Radiologists }(n=25)\end{array}$ & $\begin{array}{l}\text { Identified by at least one } \\
\text { Radiologist }(n=25)\end{array}$ & $\mathrm{Ri}$ & $\begin{array}{l}95 \% \\
\mathrm{Cl}\end{array}$ \\
\hline $\begin{array}{l}\text { Bibasilar PL Nodules (= or } \\
4 \mathrm{~mm} \text { ) }\end{array}$ & 10 & 14 & 0.74 & $\begin{array}{l}0.50 \\
- \\
0.88\end{array}$ \\
\hline Bilateral PL Nodules Other & 2 & 7 & 0.38 & $\begin{array}{l}0- \\
0.66\end{array}$ \\
\hline $\begin{array}{l}\text { Bilateral PL Nodularity } \\
(<4 \mathrm{~mm})\end{array}$ & 0 & 1 & 1 & \\
\hline $\begin{array}{l}\text { Nodules surrounding } \\
\text { airways }\end{array}$ & 8 & 16 & 0.41 & $\begin{array}{l}0.04 \\
-0.69\end{array}$ \\
\hline Septal thickening in bases & 3 & 13 & 0.37 & -0.68 \\
\hline Splenomegaly/Splenectomy & 17 & 20 & 0.73 & $\begin{array}{l}0.49 \\
0.87\end{array}$ \\
\hline LAN & 7 & 13 & 0.39 & $\begin{array}{l}0.17 \\
- \\
0.67\end{array}$ \\
\hline Bilateral GGO/Cysts & 0 & 3 & -0.06 & -0.76 \\
\hline Overall & & & 0.76 & $\begin{array}{l}0.53- \\
0.89\end{array}$ \\
\hline GLILD Diagnosis & Radiologist 1 & Radiologists 2 & $\begin{array}{l}\text { Final } \\
\text { Scoring }\end{array}$ & \\
\hline High Confidence & 11 & 9 & 12 & \\
\hline Intermediate Confidence & 1 & 7 & 3 & \\
\hline Low Confidence & 13 & 9 & 10 & \\
\hline
\end{tabular}

PL=perilymphatic, LAN=lymphadenopathy, GGO=ground glass opacites

\section{Figures}



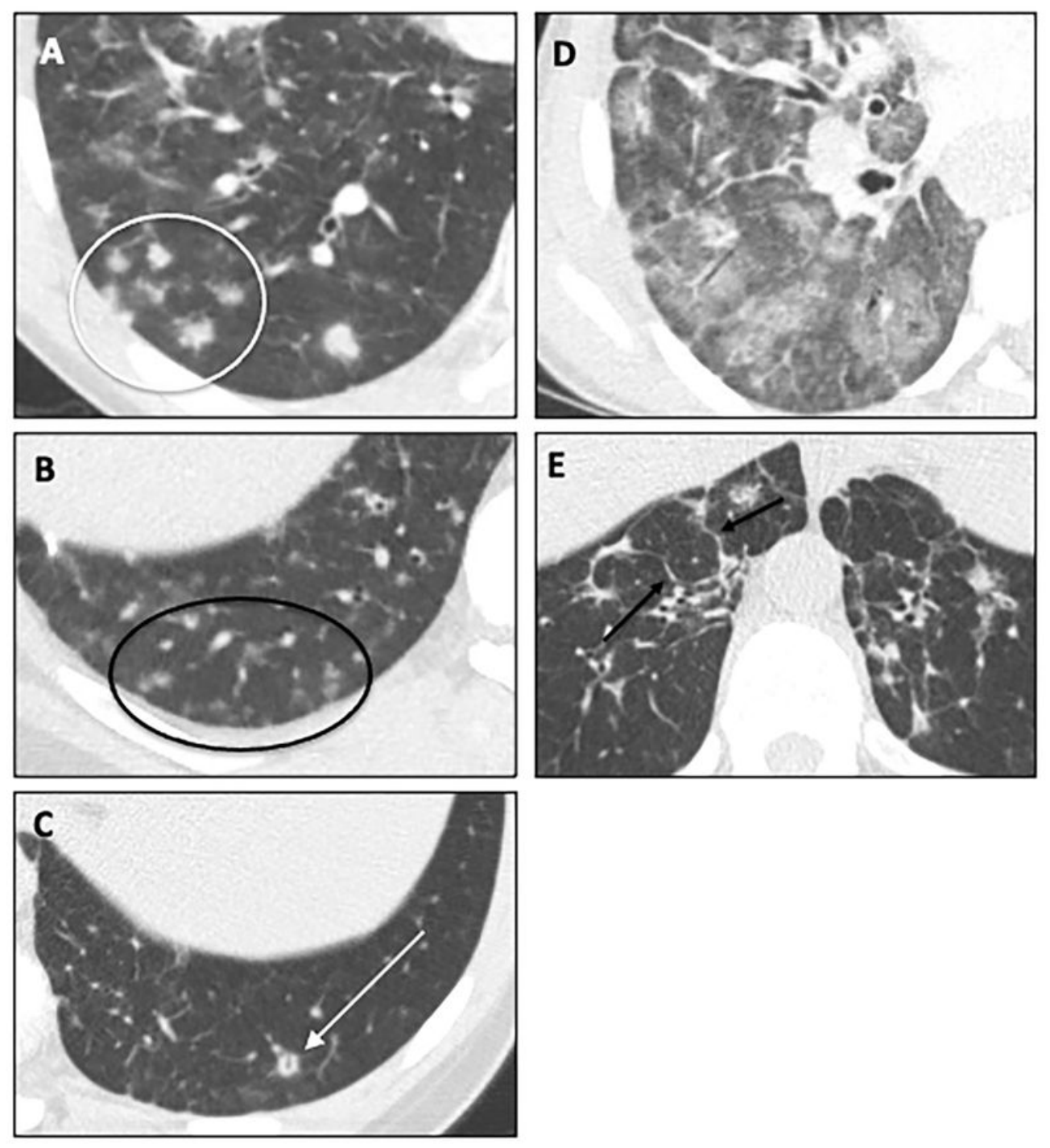

\section{Figure 1}

Representative images of pulmonary characteristics used in the GLILD CT scoring system: (A) Basilar predominant perilymphatic nodules measuring $4 \mathrm{~mm}$ or greater, circled in white, (B) Basilar predominant perlymphatic nodularity measuring less than $4 \mathrm{~mm}$, circled in black, (C) Pulmonary nodules surrounding a patent airway, white arrow, (D) Ground-glass opacities throughout the visualized lung, (E) Septal thickening, black arrows. No cases demonstrated cystic lung disease. 


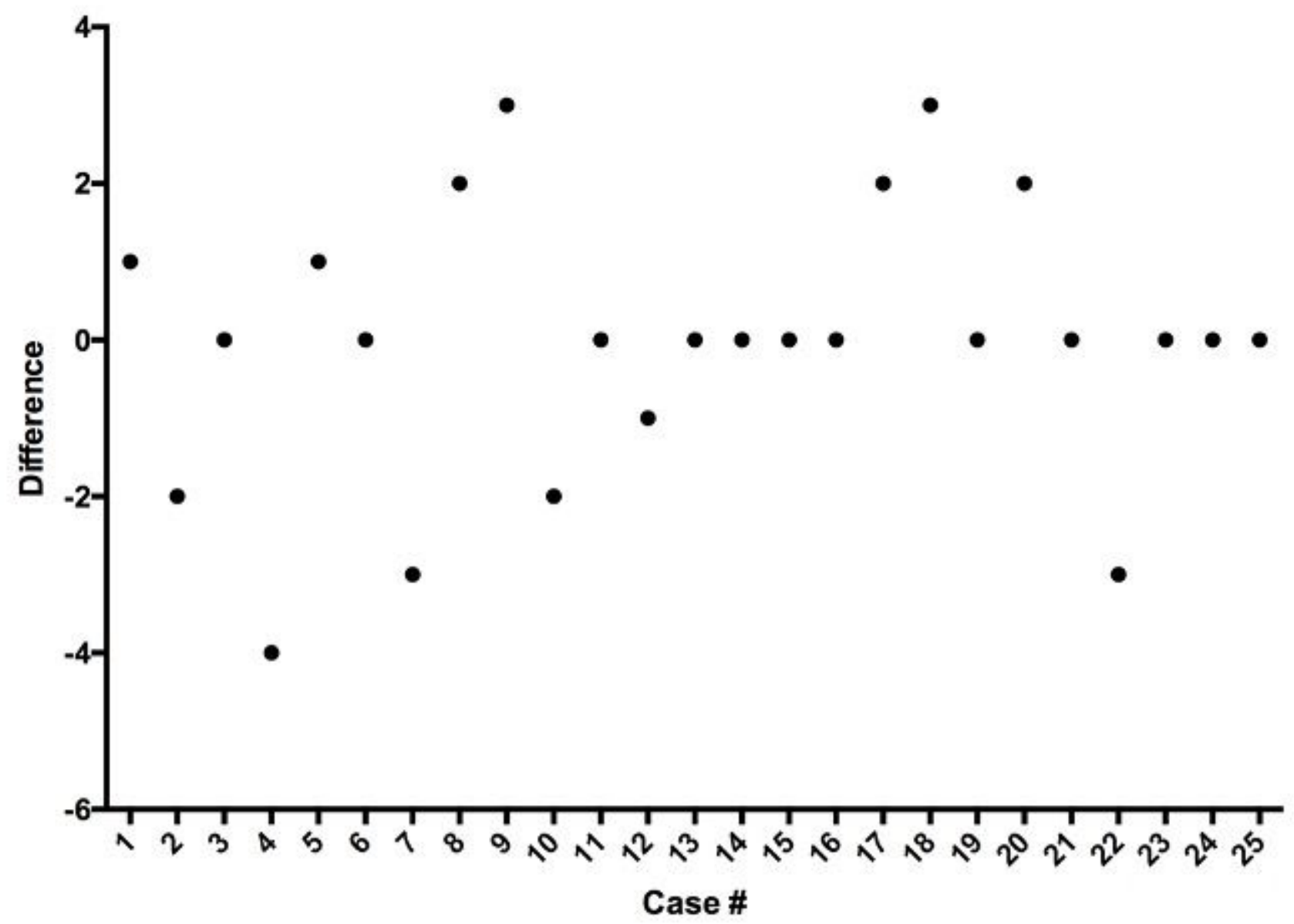

Figure 2

Bland Altmann Plot showing agreement between observers for each case.

\section{Supplementary Files}

This is a list of supplementary files associated with this preprint. Click to download.

- COIDisclosureFormSH.pdf 